\title{
The enhanced expression of IL-17-secreting T cells during the early progression of atherosclerosis in ApoE-deficient mice fed on a western-type diet
}

\author{
Un Sil Jeon ${ }^{1}$, Jun-Pyo Choi ${ }^{2}$, You-Sun $\mathrm{Kim}^{3}$, Sung-Ho Ryu ${ }^{1}$ and Yoon-Keun Kim²
}

\begin{abstract}
Atherosclerosis is a chronic progressive inflammatory disorder and the leading cause of cardiovascular mortality. Here we assessed the dynamic changes of T-cell-derived cytokines, such as inteferon (IFN)- $\gamma$, interleukin (IL)-17 and IL-4, during the progression of atherosclerosis in apolipoprotein E-null $\left(\mathrm{ApoE}^{-I-}\right)$ mice, to understand the role of immune responses in different stages of atherosclerosis. Male ApoE-/- mice were fed a high-fat, western-type diet (WD: $21 \%$ lipid, $1.5 \%$ cholesterol) after 5 weeks of age and were compared with C57BL/6 wild-type control mice fed a standard chow diet. Atherosclerotic lesions appeared in the aortic sinus of $\mathrm{ApoE}^{-/-}$mice 4 weeks after WD and the lesions progressed and occupied $>50 \%$ of the total sinus area 16 weeks after WD. Aortic IL-17 mRNA and protein expression started to increase in ApoE $^{-/-}$mice after 4 weeks on the WD and peaked at around 8-12 weeks on the WD. In terms of systemic expression of T-cell-derived cytokines, IL-17 production from splenocytes after anti-CD3/CD28 stimuli increased from 4 weeks on the WD, peaked at 12 weeks and returned to control levels at 16 weeks. The production of IFN- $\gamma$ and IL-4 (Th1 and Th2 cytokines, respectively) from splenocytes was delayed compared with IL-17. Taken together, the present data indicate that Th17 cell response may be involved at an early stage in the development of atherosclerosis.
\end{abstract}

Experimental \& Molecular Medicine (2015) 47, e163; doi:10.1038/emm.2015.19; published online 15 May 2015

\section{INTRODUCTION}

Atherosclerosis is a leading cause of cardiovascular disease and death worldwide. It is now known as a chronic progressive immune-mediated and inflammatory disorder involving large and medium-sized arteries. ${ }^{1}$ Early atherosclerotic lesions are characterized by the accumulation of fatty streaks consisting of abnormal lipids, lipid-laden foam cells and $\mathrm{T}$ cells in the vessel wall intima. With disease progression, more complicated inflammatory processes are involved and atherosclerotic plaques are covered with fibrous caps form in the aortic vessel walls. As they grow, plaques can narrow vascular lumens and cause ischemic tissue injury. If an atherosclerotic plaque ruptures, intravascular thrombosis can be catastrophic and lead to fatal myocardial or cerebral infarction.

It is well known that both innate and adaptive immune responses are involved in the pathogenesis of atherosclerosis. ${ }^{1}$ Macrophages have been known to have important roles in the initiation and progression of atherosclerosis by forming foam cells, the main component of fatty streaks, and by secreting various inflammatory mediators. ${ }^{2} \mathrm{~T}$ cells, especially $\mathrm{CD} 4^{+}$ $\mathrm{T}$ cells, are found in both human and rodent atherosclerotic lesions. ${ }^{1} \mathrm{CD} 4+\mathrm{T}$ cells were classically subdivided into type 1 (Th1) and type 2 (Th2) cells according to their cytokine expression patterns. Th1 cells secret interferon (IFN)- $\gamma$ as the major cytokine and have been shown to promote atherosclerosis in animal models and human studies. ${ }^{3}$ Recently, Th17 cells were identified as a distinct subset of $\mathrm{CD} 4^{+} \mathrm{T}$ cells and were shown to secret interleukin (IL)-17 as their main cytokine, as well as IL-6, CXCL-1, metalloproteinases, IL-21 and IL-22. ${ }^{4,5}$ Th17 cells are known to be important in various autoimmune diseases, including multiple sclerosis, rheumatoid arthritis and systemic lupus erythematosus, which were previously regarded as Th1-mediated diseases. ${ }^{5-8}$

The pathogenic role of Th17 cells in the atherosclerosis pathogenesis has been suggested in several studies. ${ }^{9}$ However, data on the role of IL-17 in the atherosclerosis pathogenesis are contradictory in apolipoprotein E-null $\left(\mathrm{ApoE}^{-/-}\right)$mice. ${ }^{10-14}$ In the present study we aimed to investigate the expression of

\footnotetext{
${ }^{1}$ Division of Molecular and Life Science, Department of Life Sciences, Pohang University of Science and Technology (POSTECH), Pohang, Republic of Korea; ${ }^{2}$ Institute of Convergence Medicine, Ewha Womans University School of Medicine, Seoul, Republic of Korea and ${ }^{3}$ Department of Internal Medicine, Asan Medical Center, Seoul, Republic of Korea

Correspondence: Professor Y-K Kim, Ewha Institute of Convergence Medicine, Ewha Womans University Medical Center, Ewha Womans University School of Medicine, Ewha Modong Hospital, Seoul 110-744, Republic of Korea.

E-mail: juinea@ewha.ac.kr
}

Received 12 September 2014; revised 2 December 2014; accepted 7 January 2015 
cytokines derived from T-cell subsets (Th1, Th17 and Th2 cells) during the progression of atherosclerosis in $\mathrm{ApoE}^{-/-}$ mice fed a western-type diet (WD).

\section{MATERIALS AND METHODS}

\section{Animals and study protocol}

All live animal protocols were approved by the Pohang University of Science and Technology Ethics Committee. Wild-type (WT) and $\mathrm{ApoE}^{-1-}$ mice on a C57BL/6 background were purchased from The Jackson Laboratory (Bar Harbor, ME, USA) and bred in specific pathogen-free conditions at the POSTCH Biotech Center. From 5 weeks of age, male control C57BL/6 mice were fed a standard chow and male $\mathrm{ApoE}^{-1-}$ mice were fed a WD (21\% lipid, $1.5 \%$ cholesterol) purchased from Dyets (Bethlehem, PA, USA) for a given period of time ( $0-24$ weeks). Chow and water were provided ad libitum. After 0 , $1,2,4,8,12,16,20$ and 24 weeks on standard chow or the WD, mice were killed. At least five mice were used for each group at each time point. The experimental protocol is shown in Figure 1.

\section{Evaluation of physical changes and serum lipid level}

During the experiment, body weight and blood pressure were measured once a week for the first 4 weeks and every 2-4 weeks thereafter. Blood pressure was measured by a non-invasive tail-cuff method (BP-2000 Blood Pressure Analysis System, Visitech Systems, Apex, NC, USA) after acclimation. Blood and tissue samples were taken from animals immediately after they were killed. Blood urea nitrogen levels and serum lipid levels were measured by a commercial service provider (Green Cross Reference Lab, Yongin-si, Korea).

\section{Quantitation of atherosclerosis}

C57BL/6 WT and $\mathrm{ApoE}^{-1-}$ mice were anesthetized with ketamine. Blood was collected from the orbital sinus and heart. Next, the mice were perfused by cardiac puncture with cold phosphate-buffered saline to wash out blood from the heart and all vessels. ${ }^{13}$ The whole aorta from the thoracic to the abdominal aorta, including the common iliac artery, was dissected under a stereo-microscope (as described below) and the heart was removed. The surrounding fat and connective tissue were removed carefully from the aorta when the aorta was dissected. The part of the heart just below the atria, including the aortic root and the base of the heart, was obtained, embedded vertically in optimum cutting temperature medium and stored in liquid nitrogen. After trimming, sequential frozen sections (thickness, $6 \mu \mathrm{m}$ ) were cut from the point at which the three aortic valve leaflets of the aortic root emerge. For quantitation of atherosclerosis, six cryosections (one per $50 \mu \mathrm{m}$ ) were selected to cover a $300-\mu \mathrm{m}$ stretch of aorta (from the aortic sinus to the ascending aorta) and were stained with Oil Red O and counterstained with hematoxylin and eosin, as described previously..$^{15}$ Images were scanned and analyzed with the analysIS software (Olympus Life and Material Science, Hamburg, Germany). Atherosclerotic area was determined as the percentage of the crosssectional area of the aortic root that was stained by Oil Red O.

\section{Reverse transcriptase-PCR analysis}

Under a dissection microscope, the whole aorta was dissected carefully by removing the adipose tissue and collecting all the layers of the aorta. Aortic tissues were homogenized in TRIzol (Invitrogen, Carlsbad, CA, USA), according to the manufacturer's instructions. Total RNA was extracted from the aorta and $1 \mu \mathrm{g}$ of total RNA was reverse transcribed using oligo-d(T) primers and AMV-RT Taq polymerase (Promega, Madison, WI, USA), according to the manufacturer's instructions. Real-time PCR was performed using Assay-onDemand TaqMan probes and primers specific for chemokine receptor-2 (CCR2), IL-17, IL-12p40, IL-6 and GAPDH (Applied Biosystems, Foster City, CA, USA) and an ABI PRISM 7500 Sequence Detection System. The level of mRNA expression was normalized with respect to the level of GAPDH mRNA expression.

\section{Immunoblot analysis}

Aortic tissues were homogenized in cold lysis buffer $(10 \mathrm{~mm}$ Tris ( $\mathrm{pH}$ 7.5), $1 \mathrm{~mm}$ sodium orthovanadate, 1\% SDS). Protein concentration was measured by the bicinchoninic acid method (Bio-Rad, Hercules, CA, USA). Homogenate samples were run on $10 \%$ SDS-polyacrylamide gels and stained with Coomassie blue. Gel images were scanned and quantified as described below, to adjust for equal loading. After correction of protein concentrations, equal amounts of protein were run on $10 \%$ SDS-polyacrylamide gels and electroblotted onto a polyvinylidene difluoride membrane (Millipore, Bedford, MA, USA). The blots were blocked with 5\% non-fat milk in PBS-T ( $80 \mathrm{~mm}$ $\mathrm{Na}_{2} \mathrm{HPO}_{4}, 20 \mathrm{~mm} \mathrm{NaH} \mathrm{PO}_{4}, 100 \mathrm{~mm} \mathrm{NaCl}, 0.1 \%$ Tween $20 \mathrm{pH} 7.5$ ) for $30 \mathrm{~min}$ at room temperature and then incubated overnight at $4{ }^{\circ} \mathrm{C}$ with primary antibody (anti-ICAM-1 (Cell Signaling Technology, Boston, MA, USA) or anti-IL-17 (Santa Cruz Biotechnology, Santa Cruz, CA, USA). After washing, the blots were incubated at room temperature for $1 \mathrm{~h}$ with horseradish peroxidase-conjugated antirabbit IgG (Cell Signaling Technology) diluted 1:5000 in blocking

\section{Experiment Protocols \\ - Five week old apoE KO mice started to be fed a western diet (WD: lipid 20\%, cholesterol $1.5 \%$ ) \\ - Five week old C57BL/6 mice started to be fed a regular chow diet

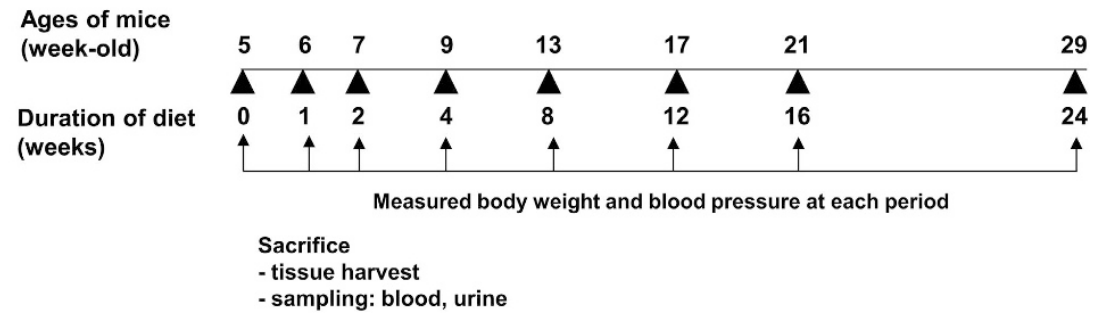

Figure 1 An experimental protocol for the generation of atherosclerosis. Male C57BL/6 and ApoE ${ }^{-1-}$ mice on a C57BL/6 background were used. From 5 weeks of age, control C57BL/6 mice were fed a standard chow and ApoE ${ }^{-1-}$ mice were fed a western-type diet (WD; $21 \%$ lipid, $1.5 \%$ cholesterol). Each group at each time point contained at least five or six mice. Mice were euthanized at 0, 1, 2, 4, 8, 12, 16, 20 and 24 weeks on the WD. 
a
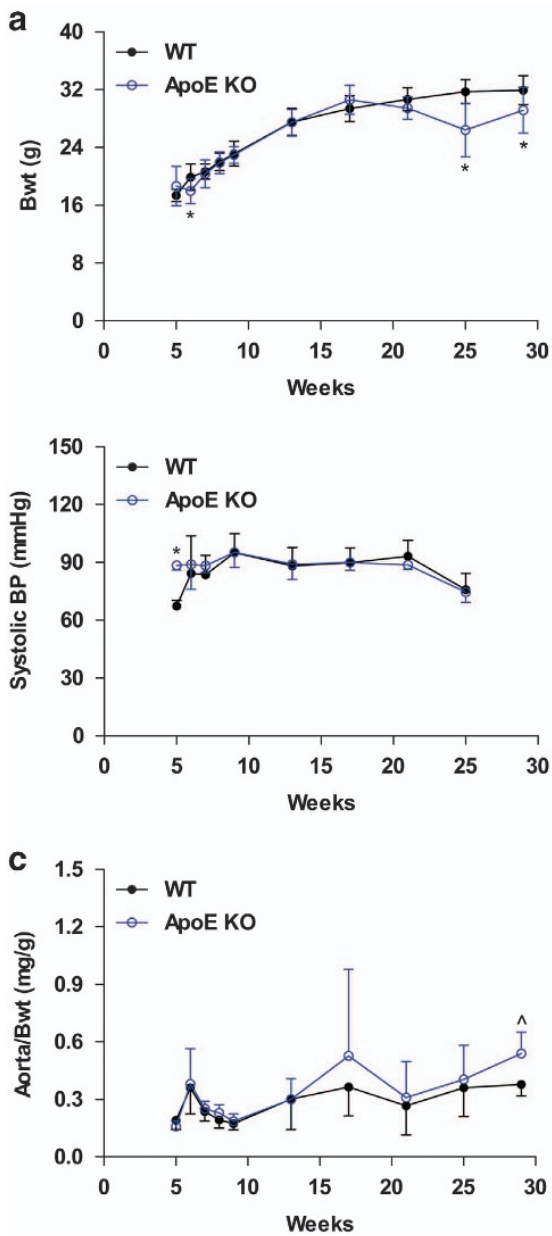
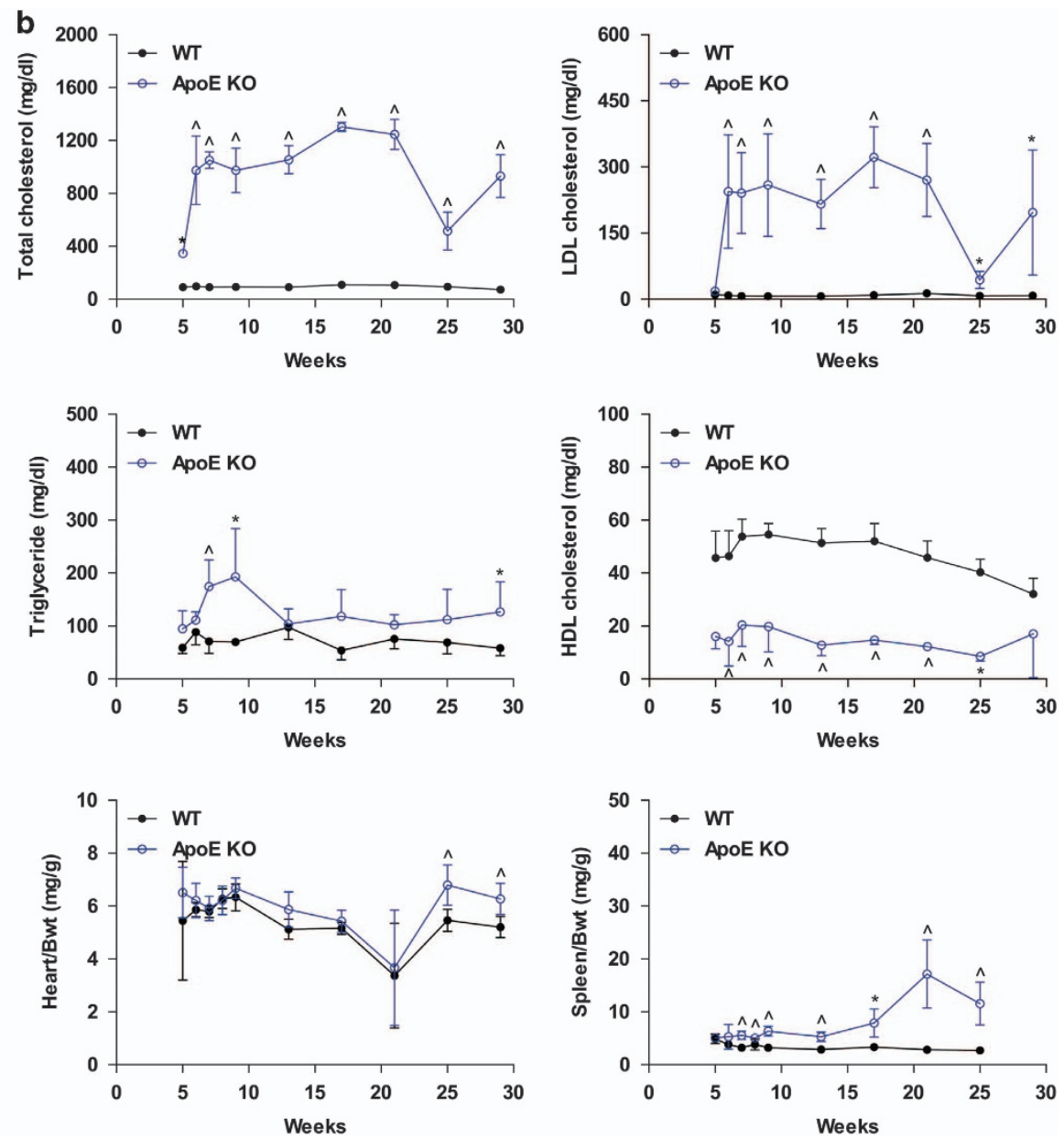

Figure 2 Kinetics of physiologic and lipid parameters of atherosclerosis. (a) The change of body weight and blood pressure during experiments. (b) Serum lipid levels. (c) Tissue weight-to-body weight ratio. ${ }^{*} P<0.05$ versus $W T, \wedge P<0.01$ versus WT.

solution. Antibody binding was visualized using the enhanced chemiluminescence system (Amersham Bioscience, Buckinghamshire, UK). Specific bands were scanned and quantified using the Quantity One software (Bio-Rad).

\section{Intracellular cytokine staining}

Splenocytes were obtained from mice and incubated at $37^{\circ} \mathrm{C}$ for $6 \mathrm{~h}$ in 48-well plates coated with anti-CD3 and anti-CD28 antibodies ( $1 \mathrm{mg} \mathrm{ml}^{-1}$ each; eBioscience, San Diego, CA, USA). Cells were stained for $30 \mathrm{~min}$ at $4{ }^{\circ} \mathrm{C}$ with surface-specific antibodies (antiCD3-allophycocyanin, anti-CD4-FITC and anti-CD8-PE-Cy5; BD Biosciences, Franklin Lakes, NJ, USA) and then fixed and permeablized through incubation for $10 \mathrm{~min}$ in $4 \%$ paraformaldehyde at room temperature. Cells were incubated for $30 \mathrm{~min}$ at room temperature with antibodies specific for candidate cytokines (anti-IFN- $\gamma-$ PE, -IL-17-PE, -IL-4-PE and -IL-10-PE; BD Biosciences) and then analyzed on a FACSCalibur flow cytometer (BD Biosciences) using the CellQuest software (CellQuest, Largo, FL, USA).

\section{Enzyme-linked immunosorbent assay for cytokine quantification}

Serum was collected when animals were killed. For T-cell re-stimulation, $4.0 \times 10^{6}$ splenocytes per $\mathrm{ml}$ were obtained from the spleen of each mouse and incubated with anti-CD3/28 antibodies for $72 \mathrm{~h}$. IL-6, IL-17 and IFN- $\gamma$ levels in supernatants or sera were measured by enzyme-linked immunosorbent assay, according to the manufacturer's instructions (R\&D Systems, Minneapolis, MN, USA).

\section{Statistical analysis}

All values are presented as the means \pm s.e.m. Groups were compared by non-parametric Mann-Whitney test and statistical significance was defined as a $P$-value $<0.05$.

\section{RESULTS}

Changes in physiologic and lipid profiles

Body weight and blood pressure were measured weekly for the first 4 weeks and then once every 2 or 4 weeks during the experiment. There were no differences in body weight or blood pressure between $\mathrm{C} 57 \mathrm{BL} / 6$ mice on standard chow and $\mathrm{ApoE}^{-1-}$ mice on the WD (Figure 2a). When animals were killed, serum was collected and blood urea nitrogen, serum creatinine and lipid levels were analyzed. Blood urea nitrogen and creatinine levels were not different between the two groups (data not shown). At 5 weeks of age, total cholesterol levels were higher in $\mathrm{ApoE}^{-/-}$mice than in C57BL/6 mice $(91 \pm 6.8$ vs $345 \pm 3.2 \mathrm{mg} \mathrm{dl}^{-1}, P<0.05$; Figure $2 \mathrm{~b}$ ), even though all mice had been fed standard chow; however, there were no significant 


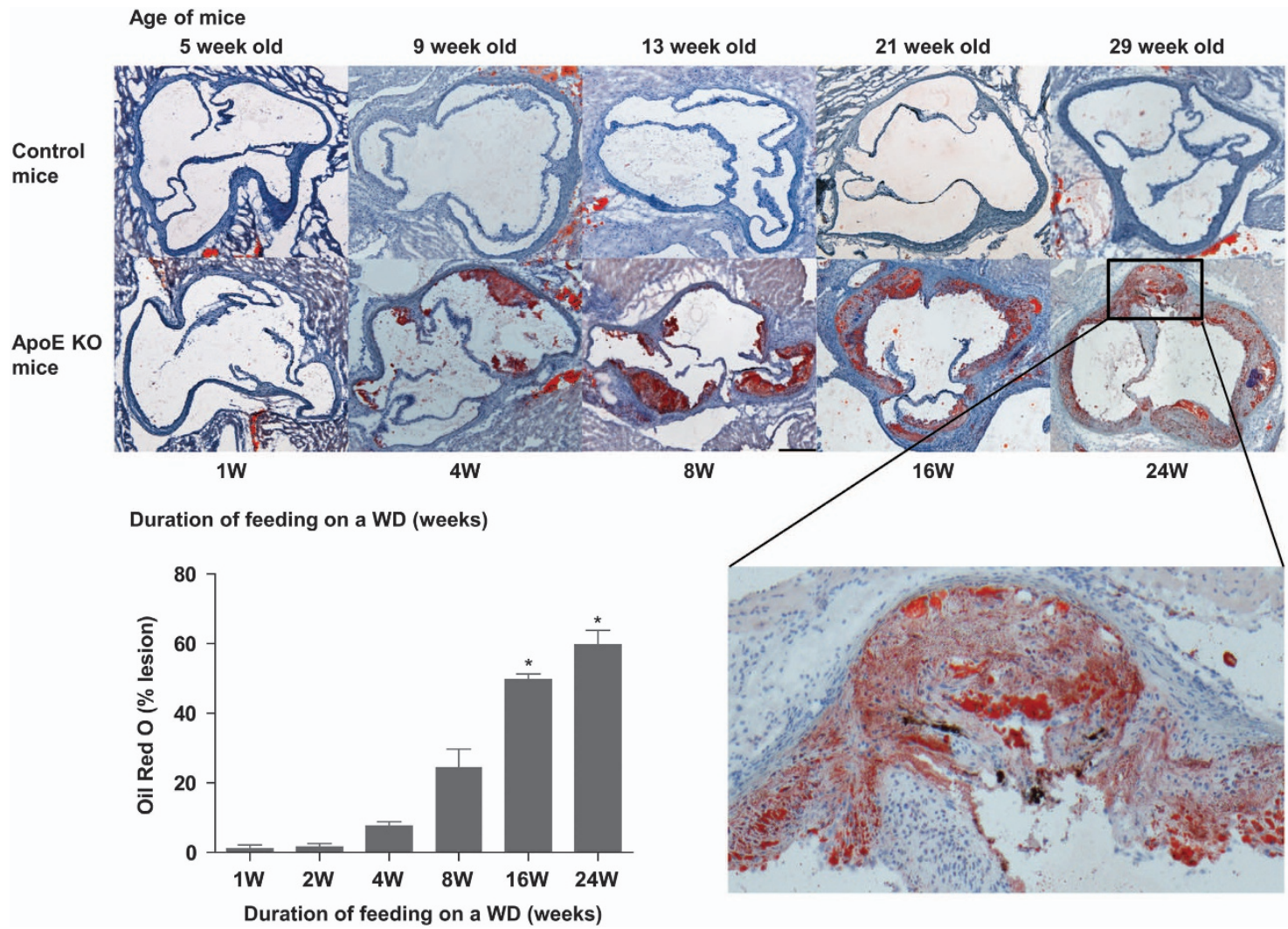

Figure 3 Photomicrographs of cross-sections of the aortic sinus of $\mathrm{ApoE}^{-/-}$mice. Aorta histologic findings. Upper panels are the results of WT mice and lower panels are ApoE-deficient mice (Oil Red $O$ and hematoxylin and eosin counter staining, $\times 100$ magnification). Enlarged panel shows accumulation of lipid in the basement of aorta when 24 weeks after high-fat diet administration. For quantitation of atherosclerotic lesions, the percentage of the total aortic sinus area stained by Oil Red 0 was determined. ${ }^{*} P<0.05$ versus WT.

differences in low-density lipoprotein (LDL)-cholesterol, triglyceride or high-density lipoprotein-cholesterol levels between these two groups. However, total cholesterol, LDL-cholesterol and triglyceride levels dramatically increased in $\mathrm{ApoE}^{-1-}$ mice with 1 week on the WD (from $345 \pm 3.2$ to $975 \pm 115.7 \mathrm{mg} \mathrm{dl}$ $\left.{ }^{-1}\right)$. High-density lipoprotein-cholesterol levels were lower in $\mathrm{ApoE}^{-1-}$ mice than in $\mathrm{C} 57 \mathrm{BL} / 6$ mice $(P<0.05)$ and were maintained at similar levels during the experimental period (Figure 2b). However, triglyceride levels were only higher in $\mathrm{ApoE}^{-/-}$mice for the first 12 weeks on the WD $(71 \pm 19.2$ vs $174 \pm$

$22.8 \mathrm{mg} \mathrm{dl}^{-1}, P<0.01$ at 2 weeks on the WD; $76 \pm 81$ vs $102 \pm 8.5 \mathrm{mg} \mathrm{dl}^{-1}, P>0.05$ at 16 weeks on the WD). The differences in total cholesterol and LDL-cholesterol levels between the two groups had disappeared by that time. Aorta weights and heart-to-body weight ratios were similar in C57BL/6 and $\mathrm{ApoE}^{-1-}$ mice at each time point, but spleen-tobody weight ratio was higher in $\mathrm{ApoE}^{-1-}$ mice $(P<0.05$; Figure 2c). At 5 weeks of age, spleen-to-body weight ratio was not different between C57BL/6 $\left(4.9 \pm 0.33 \mathrm{mg} \mathrm{g}^{-1}\right)$ and $\mathrm{ApoE}^{-1-}$ mice $\left(5.1 \pm 0.23 \mathrm{mg} \mathrm{g}^{-1}\right)$. The ratio increased significantly in $\mathrm{ApoE}^{-1-}$ mice, reaching a value of $17.2 \pm 2.04$ $\mathrm{mg} \mathrm{g}^{-1}$ after 20 weeks on the WD (ratio $2.9 \pm 0.11 \mathrm{mg} \mathrm{g}^{-1}$ in C57BL/6 mice at the same age). These results suggest that a systemic immune response was activated in WD-fed $\mathrm{ApoE}^{-1-}$ mice. No $\mathrm{ApoE}^{-1-}$ or WT control mice died during the experimental period (up to 30 weeks).

\section{Development and progression of atherosclerosis in WD-fed ApoE $^{-1-}$ mice}

We first observed the development and progression of atherosclerosis in $\mathrm{ApoE}^{-1-}$ mice over time. Atherosclerosis was quantified by measuring percentage Oil Red O-stained area within the aortic sinus. Until 5 weeks of age, C57BL/6 and $\mathrm{ApoE}^{-1-}$ mice were fed a standard chow. At that time, no significant atherosclerotic lesions were found in C57BL/6 or ApoE $^{-1-}$ mice (Figure 3). After 1 and 2 weeks on the WD, no fatty streaks were observed in the aortic sinuses of $\mathrm{ApoE}^{-1-}$ mice (data not shown). Oil Red O-positive lesions first appeared in the aortic sinus of $\mathrm{ApoE}^{-/-}$mice $(7.5 \%$ of the total aortic sinus area; Figure 3) after 4 weeks on the WD (age 9 weeks). The sizes and areas of the atherosclerotic plaques increased substantially over time (covering $24.6 \%$ of the total aortic sinus area at 8 weeks on the WD, $51.3 \%$ at 16 weeks and $57.7 \%$ at 24 weeks). With increasing age, vascular walls showed severe hyperplasia and the size of the atherosclerotic plaques increased, causing stenosis of the vessel lumen. Internal bleeding and thrombus were observed in some parts of plaques at advanced disease stages. In contrast, no atherosclerotic lesions were observed in control C57BL/6 mice during the 
experimental period. Thus, atherosclerotic lesions developed at very young ages in $\mathrm{ApoE}^{-1-}$ mice fed the WD and the disease progressed rapidly to advanced lesions over time.

\section{Production of pro-inflammatory mediators during the course of atherosclerosis}

As atherosclerosis is a chronic inflammatory disorder, we assessed dynamic changes in well-known mediators during the course of atherosclerosis in $\mathrm{ApoE}^{-/-}$mice. Monocyte accumulation in vascular walls is the earliest step in the formation of atherosclerotic plaques and has an important role in disease progression. ${ }^{1,2}$ We first observed changes in the expression of intracellular adhesion molecule (ICAM)-1 and CCR2, important mediators of the recruitment of monocytes to vascular walls. ${ }^{16,17}$ The level of ICAM-1 protein in the aorta was measured by immunoblotting. The expression of ICAM-1 protein was higher in $\mathrm{ApoE}^{-1-}$ mice on the WD than in control mice as early as 2 weeks on the WD (Figure 4a). The protein level peaked at 12 weeks on the WD and was maintained at a higher level in $\mathrm{ApoE}^{-1-}$ mice, even though the fold increase was reduced at later stages. The expression of CCR2 mRNA in the aorta was higher in $\mathrm{ApoE}^{-1-}$ mice than in control mice from experimental week 2 (7 weeks of age) (Figure $4 \mathrm{~b}$ ). The change in CCR2 mRNA expression showed a very similar pattern to that of ICAM-1 protein, but the fold increase in CCR2 mRNA expression in $\mathrm{ApoE}^{-1-}$ mice peaked slightly earlier than that of ICAM-1 protein (Figure 4b).

IL-6 is an important regulator of immune responses and is known to be involved in the pathogenesis of atherosclerosis. ${ }^{3,18}$ In addition, IL-6 is a key promoter of Th17 cell differentiation. ${ }^{4,19}$ In the current study, we assessed dynamic changes in aortic IL-6 expression during the course of atherosclerosis. The expression of IL-6 mRNA increased in the aorta of $\mathrm{ApoE}^{-1-}$ mice as early as 2 weeks on the WD (7 weeks of age), similar to ICAM-1 protein and CCR2 mRNA (Figure 5a). Interestingly, the increases in these mediators occurred before any atherosclerotic lesions were detected by microscopy. The level of IL-6 mRNA reached in $\mathrm{Apo}^{-1-}$ mice peaked after 12 weeks on the WD (116-fold increase compared to the level in WT mice). Serum IL-6 protein (measured by enzyme-linked immunosorbent assay) showed the tendency of increase in $\mathrm{ApoE}^{-1-}$ mice on the WD, but not in WT mice (Figure 5b). Serum levels of IL-6 in $\mathrm{ApoE}^{-1-}$ mice were 9.5 $\mathrm{pg} \mathrm{ml}^{-1}$ at 4 weeks on the WD, $53.3 \mathrm{pg} \mathrm{ml}^{-1}$ at 16 weeks and $18.2 \mathrm{pg} \mathrm{ml}^{-1}$ at 12 weeks. We could not calculate $P$-values of serum IL-6 levels for comparison between WT and $\mathrm{ApoE}^{-1-}$ mice, except 16 weeks data, because IL- 6 was not detected in WT mice. The data showed that serum IL-6 levels increased in
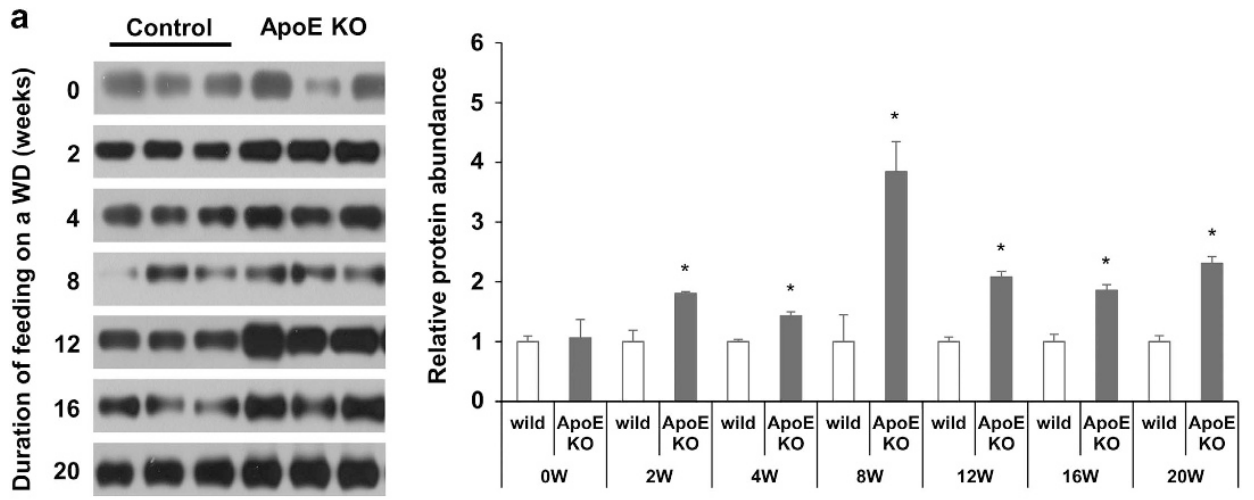

Duration of feeding on a WD (weeks)

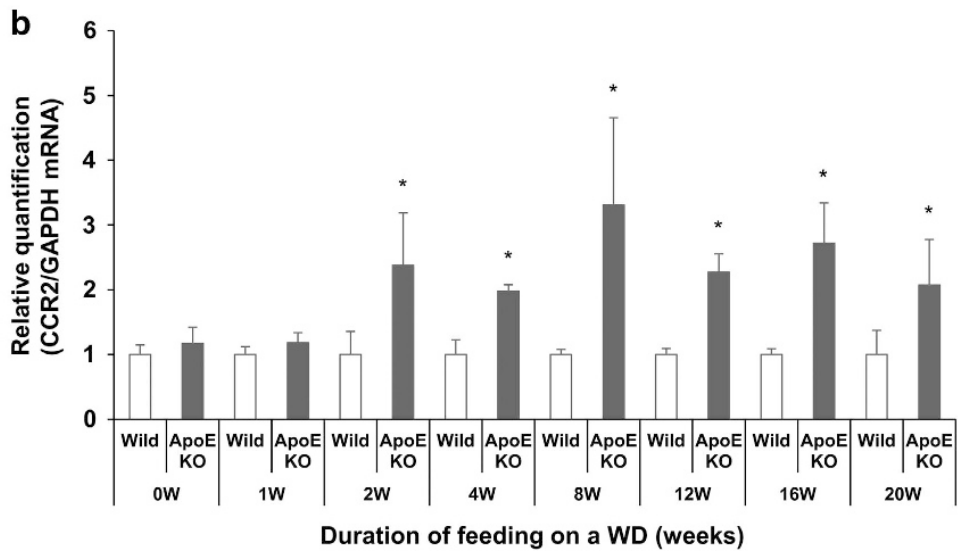

Figure 4 Kinetic changes in pro-inflammatory mediator expression in $\mathrm{ApoE}^{-1-}$ and control mice. For all figures, evaluation were done in various time point; $y$ axis: relative ratio of ICAM-1 or CCR2 levels in ApoE ${ }^{-1-}$ mice to that in WT C57BL/6 mice. ${ }^{*} P<0.05$ versus WT. (a) The immunoblotting results of ICAM-1 protein expression in aorta tissue. Left: result of immunobloting film; right: quantification results by band scanning. (b) The change of CCR2 mRNA expression level in aorta tissue. 

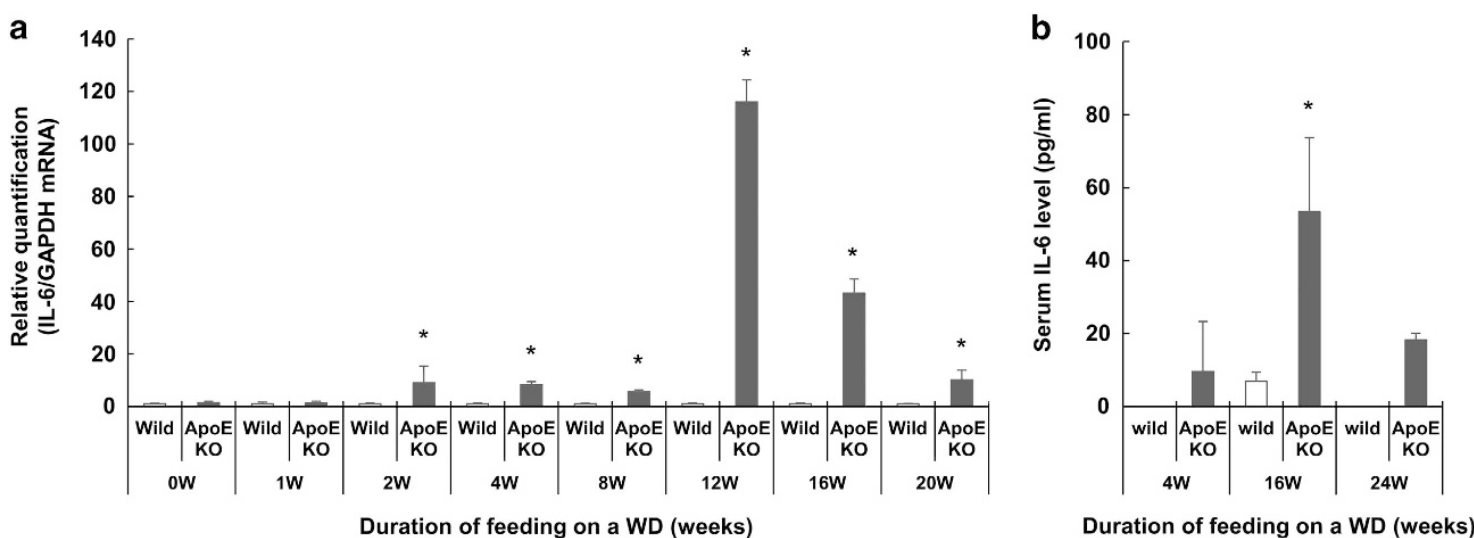

Figure 5 Kinetic changes in IL-6 expression in $\mathrm{ApoE}^{-1-}$ and control mice. For all figures, $y$-axis: relative ratio of IL-6 level in ApoE ${ }^{-1-}$ mice to that in control C57BL/6 mice. ${ }^{*} P<0.05$ versus WT. (a) The expression level of IL-6 mRNA in aorta tissue following WD administration for 24 weeks. (b) The expression of IL-6 protein in serum on each time point.

$\mathrm{ApoE}^{-1-}$ mice with the development of atherosclerosis. However, there was no direct correlation between the serum IL-6 levels and aortic expression of IL-6 mRNA (data not shown).

\section{Kinetic changes in IL-17 and IFN- $\gamma$ expression in aorta tissues}

The role of IL-17 in atherosclerosis remains unclear. Some studies showed that IL-17 accelerated the progression of atherosclerosis in animal models, ${ }^{11,12}$ but others showed the opposite. ${ }^{14}$ We evaluated serial changes in IL-17 expression in the aorta according to the development and progression of atherosclerosis over time. Total RNA and protein were obtained from the aortas of mice. The expression of IL-17 mRNA was measured by real-time quantitative PCR. At 5 weeks of age, the expression of IL-17 mRNA was very low and undetectable in most $\mathrm{ApoE}^{-1-}$ and WT mice on standard chow. IL-17 mRNA was only detected in the aorta of $\mathrm{ApoE}^{-/-}$ mice on the WD and a few WT mice (Figure 6a, left). The level of IL-17 mRNA increased in $\mathrm{ApoE}^{-1-}$ mice after 4 weeks on the WD and peaked at 8 weeks on the WD. We could not perform statistical comparisons, because IL-17 mRNA was only detected in a few WT mice. IL-17 protein levels were measured by immunoblotting and were shown to be higher in $\mathrm{ApoE}^{-/-}$ mice compared with control mice, especially after 12 weeks on the WD (Figure 6a, right).

IFN- $\gamma$ is the main cytokine of Th1 cells and known to be involved in the pathogenesis of atherosclerosis. ${ }^{1,20} \mathrm{We}$ also measured the expression of IFN- $\gamma$ and IL-12p40 mRNA in the whole aorta by real-time quantitative PCR. Those mRNA showed patterns of change that were similar to those of IL-17 (Figure 6b).

\section{Systemic T-cell responses during the course of atherosclerosis}

Finally, systemic T-cell responses during the course of atherosclerosis were evaluated. Splenocytes were obtained from mice at the time at which they were killed and were prepared for intracellular staining of cytokines. Flow cytometry analysis was performed using anti-IL-17, anti-IL-4, anti-IL-10 and
anti-IFN- $\gamma$ antibodies. With regard to Th17 cell expression (Figure $7 \mathrm{a}$ ), the percentages of $\mathrm{CD} 4^{+} \mathrm{IL}-17^{+}$splenocytes were not different between control and $\mathrm{ApoE}^{-/-}$mice at 0 and 2 weeks on the WD. The percentage of $\mathrm{CD} 4^{+} \mathrm{IL}-17^{+}$splenocytes was higher in $\mathrm{ApoE}^{-1-}$ mice compared with $\mathrm{C} 57 \mathrm{BL} / 6$ mice after 4 weeks on the WD $(0.09 \pm 0.0$ vs $0.28 \pm 0.04$, $P<0.05)$ and remained increased until 12 weeks on the WD $(0.71 \pm 0.16$ vs $3.21 \pm 0.59, P<0.05)$. However, the increase in the percentage of $\mathrm{CD} 4^{+} \mathrm{IL}-17^{+}$splenocytes in $\mathrm{ApoE}^{-1-}$ mice was blunted after 16 weeks on the WD $(0.91 \pm 0.32$ vs $1.63 \pm 0.50, P>0.05)$. There were no differences in the numbers of $\mathrm{CD}^{+}$IFN- $\gamma^{+}$splenocytes between control and $\mathrm{ApoE}^{-1-}$ mice at the early stages of atherosclerosis (Figure 7a). At 12 weeks on the $\mathrm{WD}$, the percentage of $\mathrm{CD} 4^{+} \mathrm{IFN}-\gamma^{+}$ splenocytes was higher in $\mathrm{ApoE}^{-/-}$mice compared with control mice $(1.92 \pm 0.15$ vs $6.60 \pm 1.37, P<0.05)$. The percentage of $\mathrm{CD} 4^{+} \mathrm{IL}-4^{+}$splenocytes was not different between WD-fed $\mathrm{ApoE}^{-1-}$ and control mice at most time points, but was higher in $\mathrm{ApoE}^{-1-}$ mice at 12 weeks on the WD $(0.71 \pm 0.12$ vs $2.52 \pm 0.54, P<0.05)$. As the proportion of splenic Th1 and Th17 cells in $\mathrm{ApoE}^{-1-}$ mice on the WD peaked at 12 weeks, cytokine levels were measured in supernatants from anti-CD3/CD28-stimulated splenocytes obtained from mice at that time point. This experiment showed that the production for IL-17 was significantly increased in $\mathrm{ApoE}^{-1-}$ mice than in C57BL/6 mice, whereas that of IFN- $\gamma$ was not (Figure $7 b$ ).

\section{DISCUSSION}

In the present study we assessed the morphology and kinetic changes in IL-17 and various mediators from the initiation to the late stages of atherosclerosis in $\mathrm{ApoE}^{-1-}$ mice on a WD. We found that aortic expression of IL-17 protein and mRNA increased in $\mathrm{ApoE}^{-1-}$ mice at an early stage of atherosclerosis. In addition, expression of the inflammatory mediators CCR2, ICAM-1 and IL-6 increased in the aorta of $\mathrm{ApoE}^{-1-}$ mice before development of any morphologic lesions.

We used $\mathrm{ApoE}^{-1-}$ mice as an animal model of atherosclerosis, because atherosclerotic lesions in $\mathrm{ApoE}^{-1-}$ mice are 

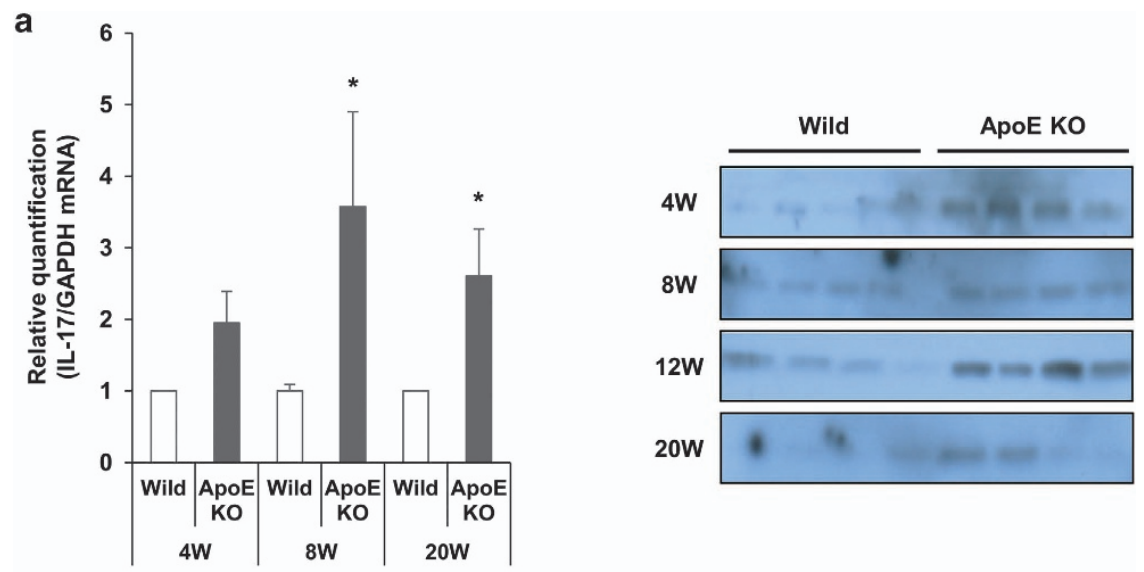

Duration of feeding on a WD (weeks)
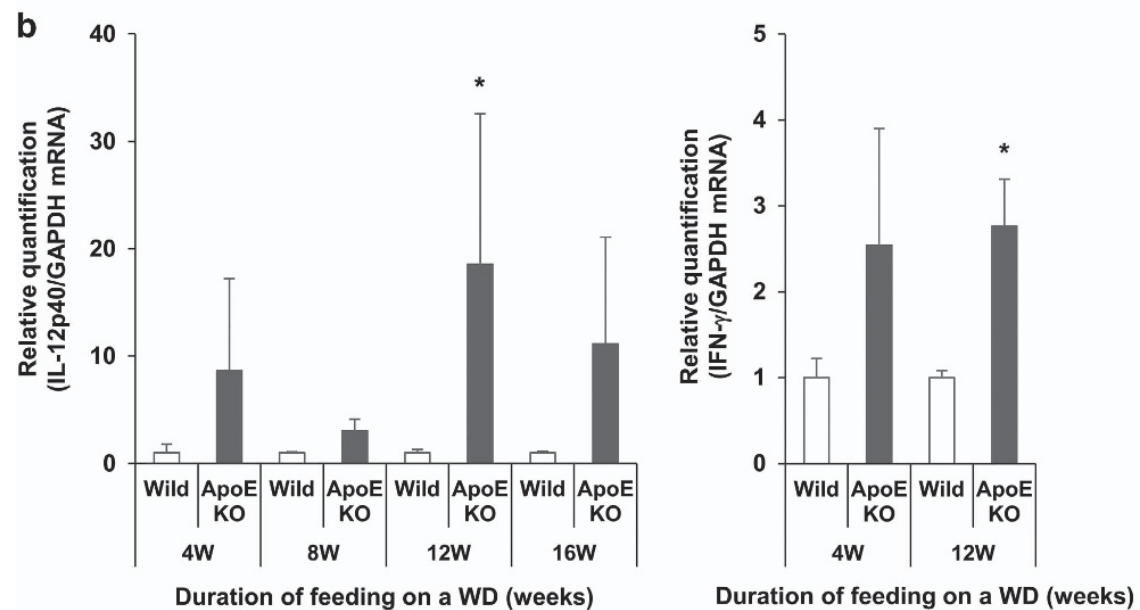

Figure 6 Kinetic changes in IL-17 expression in $\mathrm{ApoE}^{-1-}$ and control mice. For all figures, $y$-axis: relative ratio of the level in ApoE $\mathrm{E}^{-/-}$ mice to that in control C57BL/6 mice. ${ }^{*} P<0.05, \wedge P<0.01$ versus WT. (a) The expression of IL-17 mRNA and protein in aorta tissue. (left panel: result of mRNA expression; right: immunoblot analysis). (b) The expression of IFN- $\gamma$ mRNA in aorta.

known to be very similar to those in humans. ${ }^{21}$ Normally, atherosclerosis occurs spontaneously in $\mathrm{ApoE}^{-1-}$ mice, but western diets containing high levels of lipids cause atherosclerosis to occur earlier and be more severe than does a normal standard diet. ${ }^{21}$ Therefore, we compared WD-fed $\mathrm{ApoE}^{-1-}$ mice with WT mice fed a standard chow diet to make the differences between the groups more distinct. We measured atherosclerotic lesions within the aortic sinus, because this is usually the first site of development of atherosclerotic lesions in $\mathrm{ApoE}^{-/-}$mice. ${ }^{15,21}$

In our model, atherosclerotic plaques developed in the aortic sinus as early as 9 weeks of age (4 weeks on the WD), consistent with a previous report. ${ }^{15}$ The atherosclerotic lesions showed rapid progression and increased steadily in size and extent over time. Interestingly, we found that an inflammatory response preceded any visible morphologic atherosclerotic changes. At 5 weeks of age, when both WT and $\mathrm{ApoE}^{-1-}$ mice had been fed standard chow, there was no difference in the expression of ICAM-1, CCR2 or IL-6 between these two groups. However, expression of these mediators started to increase at 2 weeks in WD-fed $\mathrm{ApoE}^{-/-}$mice. In our model, serum cholesterol and LDL-cholesterol levels were higher in $\mathrm{ApoE}^{-1-}$ mice than in control mice at baseline (5 weeks of age), but dramatically increased in $\mathrm{ApoE}^{-/-}$mice after only 1 week on the WD. Considering that hyperlipidemia and subsequent formation of ox-LDL induce endothelial cell damage to initiate the inflammatory reactions, and that both ICAM-1 and CCR2 have important roles in adhesion and the recruitment of monocytes during the development of fatty streaks on vascular walls, the occurrence of inflammation before the formation of fatty streaks would be relevant. A previous study demonstrated that most pro-inflammatory mediators reached maximum levels after about 10 weeks in $\mathrm{ApoE}^{-1-}$ mice on a WD, which were consistent with our present data. ${ }^{22}$ Taken together, these data suggest that inflammatory processes are important in the development of atherosclerosis, especially at the early stages.

According to previous observations, fibrous capsule maturation on the surface of atherosclerotic plaques occurs at a late stage of atherosclerosis. ${ }^{15,22,23}$ Therefore, chronic processes such as fibrosis, acute inflammatory processes, may be the major pathologic processes at late disease stages. Despite the 

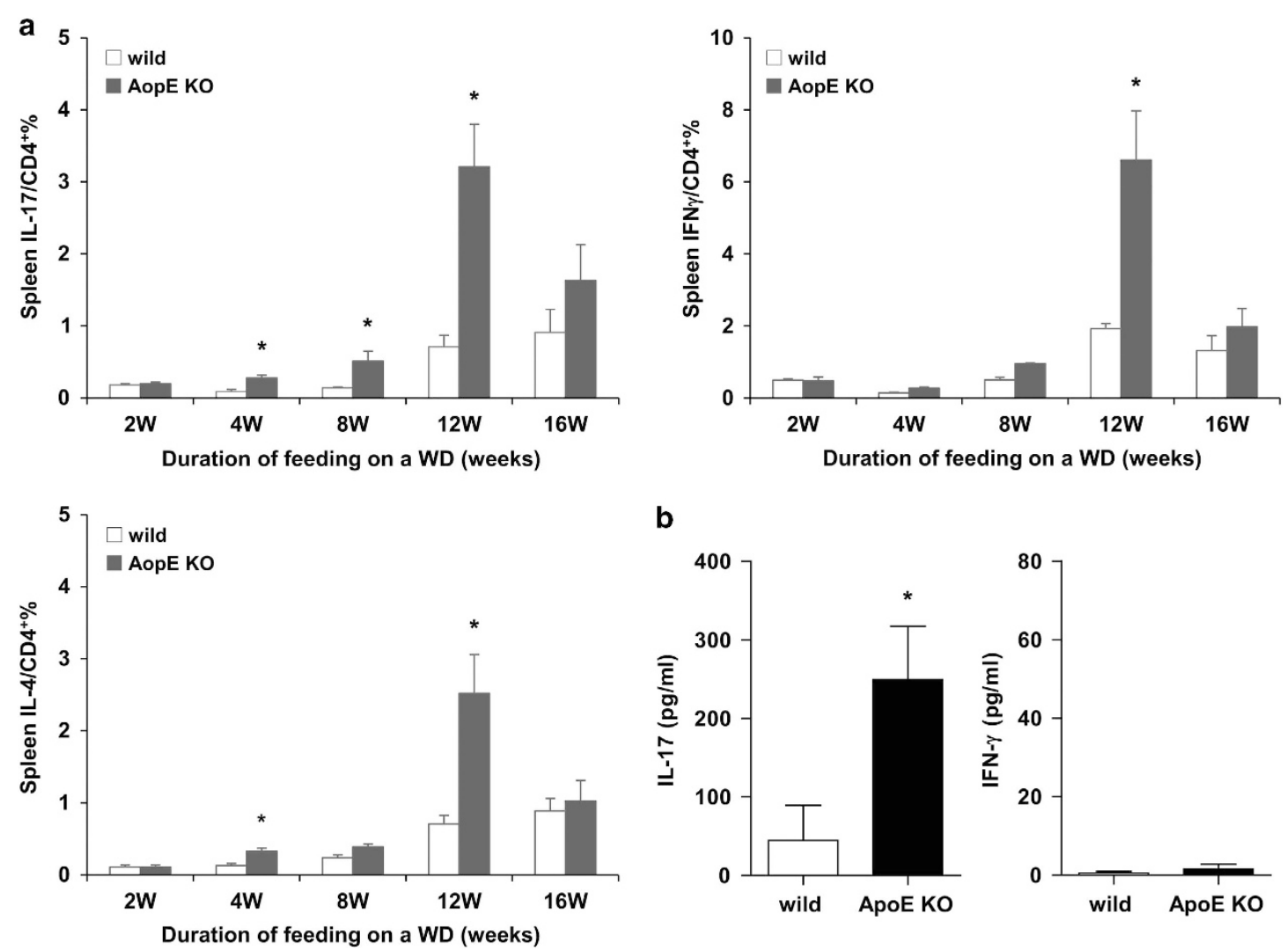

Figure 7 The systemic expression of T-cell-derived cytokines in ApoE ${ }^{-/-}$and control mice. (a) The proportion of IL-17-, IFN- $\gamma$ - and IL-4positive CD4 T cells in splenocytes. (b) The production of IL-17 and IFN- $\gamma$ from splenocytes at 12 weeks after anti-CD3/CD28 stimulation. ${ }^{*} P<0.05$ versus control.

continuous enlargement of atherosclerotic lesions over time, the present study showed that the expression of ICAM-1, CCR2 and IL-6 reached peak levels at 12-16 weeks on the WD (17-21 weeks of age), and that fold increases in $\mathrm{ApoE}^{-/-}$mice subsequently decreased. Pro-inflammatory mediators showed very similar patterns of expression during the course of atherosclerosis: (1) increased levels at a very early stage, before the development of visible atherosclerotic plaques; (2) peak levels at about 8-12 weeks on the WD; and (3) reduced fold increase at late stages of atherosclerosis. Considering the complicated machinery involved in the pathogenesis of atherosclerosis, these data suggest that the main pathogenic factor(s) in atherosclerosis progression at late disease stages may be related to tissue remodelling rather than inflammation.

It is well documented that serum IL-17 levels in atherosclerosis patients increase during acute coronary syndrome, ${ }^{9,10}$ and that IL-17 is found in atherosclerotic lesions in both humans and animals. ${ }^{1,9}$ Our data suggest that the expression of IL- 6 preceded the Th17 cell response. IL- 6 is a key mediator in the development of Th17 cell responses. ${ }^{19}$ In addition, IL-6 is known to be a downstream mediator of the effects of IL-17. ${ }^{19}$ Taken together, these findings suggest that WD-induced production of IL-6 is a key pathogenic process in the development of adaptive immune dysfunction in $\mathrm{ApoE}^{-1-}$ mice.
To our knowledge, no previous study has evaluated kinetic changes in IL-17 and related molecules during the course of atherosclerosis. We found the IL-17 levels in the aorta and spleen increased in WD-fed $\mathrm{ApoE}^{-1-}$ mice, consistent with previous reports. ${ }^{9,11}$ The expression of IL-17 in the aorta was very low or not detected in C57BL/7 mice (regardless of age) and $\mathrm{ApoE}^{-1-}$ mice at 5 weeks of age, and fed standard chow. In $\mathrm{ApoE}^{-/-}$mice, aortic expression of IL-17 started to increase after 4 weeks on the WD (9 weeks of age), the time at which visible atherosclerotic lesion appeared in the aortic sinus. Even though its expression increased slightly later than that of CCR2, IL- 6 and ICAM-1, IL-17 appears to have a role from the very early stages of atherosclerosis. Th1 cells have a key role in the pathogenesis of atherosclerosis. ${ }^{1,3}$ Therefore, we assessed kinetic changes in IFN- $\gamma$, the key cytokine of Th1 cells, and IL-12p40, a component of both IL-12 and IL-23. The expression of these cytokines was increased in WD-fed $\mathrm{ApoE}^{-/-}$mice in the aorta and splenocytes. However, the increase of IL-12p40 mRNA in the aorta and IFN- $\gamma$ in the spleen occurred later than the change in IL-17 expression. In addition, when we were evaluating the level of IL-12p70 in serum we did not confirm the expression in that time. We supposed that the reason of high Th17 immune responses is caused by low Th1 cell development through low expression of IL-12p70 in this animal model and IFN- $\gamma$-positive $\mathrm{T}$ cell-detection is caused by 'T-cell plasticity', the conversion of Th17 cell to Th1 cell, 
as increase of IL-12p40 and IFN- $\gamma$ showed the 'interval' compared with IL-17 expression and IL-12p40 is related with T-cell plasticity. ${ }^{24}$ To sum up, these data suggest that Th17 cell responses may be important in the atherosclerosis pathogenesis.

In summary, we found that IL-17 expression was increased from the very early stages of atherosclerosis, and that production of proinflammatory mediators was increased before morphologic changes occurred. However, the expression of IL-17 and proinflammatory mediators was reduced at the advanced stages of atherosclerosis. Therefore, early intervention to modulate immune dysfunction might be effective for preventing the development and progression of atherosclerosis.

\section{CONFLICT OF INTEREST}

The authors declare no conflict of interest.

\section{ACKNOWLEDGEMENTS}

We thank members of the Pohang University of Science and Technology animal facility for their experimental expertise. This study was supported by a grant from the Korea Ministry of Health and Welfare, Republic of Korea (HI 13C 0040-010014 and HI 14C 2628).

1 Hansson GK, Hermansson A. The immune system in atherosclerosis. Nat Immunol 2011; 12: 204-212.

2 Moore KJ, Tabas I. Macrophages in the pathogenesis of atherosclerosis. Cell 2011; 145: 341-355.

3 Hansson GK, Libby P. The immune response in atherosclerosis: a double-edged sword. Nat Rev Immunol 2006; 6: 508-519.

4 Harrington LE, Hatton RD, Mangan PR, Turner H, Murphy TL, Murphy KM et al. Interleukin 17-producing CD4+ effector T cells develop via a lineage distinct from the T helper type 1 and 2 lineages. Nat Immunol 2005; 6: 1123-1132.

5 Korn T, Bettelli E, Oukka M, Kuchroo VK. IL-17 and Th17 Cells. Annu Rev Immunol 2009; 27: 485-517.

6 Montes M, Zhang X, Berthelot L, Laplaud DA, Brouard S, Jin J et al. Oligoclonal myelin-reactive T-cell infiltrates derived from multiple sclerosis lesions are enriched in Th17 cells. Clin Immunol 2009; 130: 133-144.

7 Hirota K, Yoshitomi H, Hashimoto M, Maeda S, Teradaira S, Sugimoto N et al. Preferential recruitment of CCR6-expressing Th17 cells to inflamed joints via CCL20 in rheumatoid arthritis and its animal model. J Exp Med 2007; 204: 2803-2812.

8 Pernis AB. Th17 cells in rheumatoid arthritis and systemic lupus erythematosus. J Intern Med 2009; 265: 644-652.

9 Butcher M, Galkina E. Current views on the functions of interleukin-17Aproducing cells in atherosclerosis. Thromb Haemost 2011; 106: 787-795.

10 Eid RE, Rao DA, Zhou J, Lo SF, Ranjbaran H, Gallo A et al. Interleukin-17 and interferon-gamma are produced concomitantly by human coronary artery-infiltrating $\mathrm{T}$ cells and act synergistically on vascular smooth muscle cells. Circulation 2009; 119: 1424-1432.
11 Erbel C, Chen L, Bea F, Wangler S, Celik S, Lasitschka F et al. Inhibition of IL-17A attenuates atherosclerotic lesion development in apoEdeficient mice. J Immunol 2009; 183: 8167-8175.

12 Smith E, Prasad KM, Butcher M, Dobrian A, Kolls JK, Ley K et al. Blockade of interleukin-17A results in reduced atherosclerosis in apolipoprotein E-deficient mice. Circulation 2010; 121: 1746-1755.

13 Gao Q, Jiang Y, Ma T, Zhu F, Gao F, Zhang P et al. A critical function of Th17 proinflammatory cells in the development of atherosclerotic plaque in mice. J Immunol 2010; 185: 5820-5827.

14 Danzaki $K$, Matsui $Y$, Ikesue $M$, Ohta $D$, Ito $K$, Kanayama $M$ et al. Interleukin-17A deficiency accelerates unstable atherosclerotic plaque formation in apolipoprotein E-deficient mice. Arterioscler Thromb Vasc Biol 2012; 32: 273-280.

15 Nakashima Y, Plump AS, Raines EW, Breslow JL, Ross R. ApoE-deficient mice develop lesions of all phases of atherosclerosis throughout the arterial tree. Arterioscler Thromb 1994; 14: 133-140.

16 Nakashima Y, Raines EW, Plump AS, Breslow JL, Ross R. Upregulation of VCAM-1 and ICAM-1 at atherosclerosis-prone sites on the endothelium in the ApoE-deficient mouse. Arterioscler Thromb Vasc Biol 1998; 18: 842-851.

17 Charo IF, Peters W. Chemokine receptor 2 (CCR2) in atherosclerosis, infectious diseases, and regulation of T-cell polarization. Microcirculation 2003; 10: 259-264.

18 Haddy N, Sass C, Droesch S, Zaiou M, Siest G, Ponthieux A et al. IL-6, TNF-alpha and atherosclerosis risk indicators in a healthy family population: the STANISLAS cohort. Atherosclerosis 2003; 170: 277-283.

19 Kim YS, Hong SW, Choi JP, Shin TS, Moon HG, Choi EJ et al. Vascular endothelial growth factor is a key mediator in the development of $\mathrm{T}$ cell priming and its polarization to type 1 and type 17T helper cells in the airways. J Immunol 2009; 183: 5113-5120.

20 Gupta S, Pablo AM, Jiang X, Wang N, Tall AR, Schindler C. IFN-gamma potentiates atherosclerosis in ApoE knock-out mice. J Clin Invest 1997; 99: 2752-2761.

21 Meir KS, Leitersdorf E. Atherosclerosis in the apolipoprotein-E-deficient mouse: a decade of progress. Arterioscler Thromb Vasc Biol 2004; 24: 1006-1014.

22 Veillard NR, Steffens S, Burger F, Pelli G, Mach F. Differential expression patterns of proinflammatory and antiinflammatory mediators during atherogenesis in mice. Arterioscler Thromb Vasc Biol 2004; 24: 2339-2344.

23 Reddick RL, Zhang SH, Maeda N. Atherosclerosis in mice lacking apo E. Evaluation of lesional development and progression. Arterioscler Thromb 1994; 14: 141-147.

24 Lee YK, Turner H, Maynard CL, Oliver JR, Chen D, Elson CO et al. Late developmental plasticity in the T helper 17 lineage. Immunity 2009; 30: 92-107.

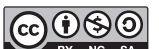

This work is licensed under a Creative Commons Attribution-NonCommercial-ShareAlike 4.0 International License. The images or other third party material in this article are included in the article's Creative Commons license, unless indicated otherwise in the credit line; if the material is not included under the Creative Commons license, users will need to obtain permission from the license holder to reproduce the material. To view a copy of this license, visit http:// creativecommons.org/licenses/by-nc-sa/4.0/ 